\title{
ON THE ASYMPTOTIC BEHAVIOR OF A FUNDAMENTAL SET OF SOLUTIONS
}

BY

\author{
CHARLES POWDER
}

\begin{abstract}
We consider $\boldsymbol{n}$ th order homogeneous linear ordinary differential equations whose coefficients have an asymptotic expansion as $x \rightarrow \infty$ in terms of real powers of $x$ and are analytic in sectors of the complex plane. In earlier work Bank (Funkcial. Ekvac. 11 (1968), 87-100) developed a method for reading off the asymptotic behavior of solutions directly from the equation, except in certain cases where roots asymptotically coalesce. For our results, we consider coefficients in a field of the type developed by Strodt (Trans. Amer. Math. Soc. 105 (1962), 229-250). By successive algebraic transforms, we show that an equation in the exceptional case can be reduced to the nonexceptional case and so the asymptotic behavior of the solutions can be read from the equation. This generalizes the classical results when $\infty$ is a singular point and the coefficients are analytic in neighborhoods of $\infty$. The strength of our results is that the coefficients need not be defined in a full neighborhood of $\infty$, and that the asymptotic behavior can be read directly from the equation.
\end{abstract}

1. Introduction. In the classical theory of ordinary differential equations, the problem of singular points has been approached in several ways. In this paper we take the approach of S. Bank in [1], [4] and [5]. In [1] and [5], Bank developed a unified theory which included both the case of the regular singular point and the case of the irregular singular point which we take here to be $\infty$. He considered equations of the form $\sum_{j=0}^{n} f_{j}(x) y^{(n-j)}=0$, where each $f_{j}(x)$ is defined in and analytic in a sector

$$
a<\arg (x-\beta \exp (i(a+b) / 2))<b
$$

(for fixed $a$ and $b$ in $(-\pi, \pi)$ and some $\beta \geqslant 0$ ), and has an asymptotic expansion as $x \rightarrow \infty$ in terms of real (not necessarily integral) powers of $x$ and/or trivial functions (functions which are $o\left(x^{a}\right)$ for all real $a$ ). Such coefficients need not be defined in a full neighborhood of $\infty$.

K. Takahasi [8] considered the problem for systems using different techniques. His results are similar; however, one cannot read the asymptotic behavior directly from the equation as in the differential algebraic techniques developed by Bank and used here for $n$th order equations.

Presented to the Society, January 25, 1979; received by the editors October 26, 1977.

AMS (MOS) subject classifications (1970). Primary 34A20, 34D05, 34E05.

Key words and phrases. Fundamental set of solutions, representation of solutions, asymptotic behavior of solutions. 
In [1] it was shown that a certain algebraic polynomial $F(\alpha)$ in $\alpha$ alone, whose degree $p$ is called the critical degree, leads to $p$ solutions of the equation of the form $y \sim x^{\alpha}(\log x)^{\beta}$, where $\alpha$ is a complex number and $\beta$ is a nonnegative integer. When $p=n$, the result is an asymptotic analog of the Fuchs regularity theorem. The result is summarized in $\$ 4$.

Later in [5], Bank studied another algebraic polynomial $H(w)$, whose coefficients are functions of $x$ and whose degree is $n-p$. It was shown in [5] that, when the roots of $H$ are asymptotically distinct, there exist $n-p$ solutions of the form $\exp \int^{x} V$, where $V$ is asymptotically equivalent to a root of $H$. Thus the equation has a fundamental set of solutions $p$ of which are of the first type and $n-p$ of which are of the latter type. Both polynomials $F$ and $H$ can be read directly from the equation. There is also an algorithm [2, Theorem II] to read off the asymptotic behavior of the roots of $H$.

For the case $n=2$, the restriction that the roots be asymptotically distinct was removed in [4] when the coefficients lie in a certain type of function field. In [4], Bank transformed the equation (under the change of variable $y=e^{z}$, $\left.w=x z^{\prime}\right)$ to a first-order Ricatti equation. Because of the field condition, this equation could be further transformed into one for which the first-order existence theorem of Strodt $[6, \S 127]$ was applicable. However, for general $n$, the same transformation would only reduce the $n$th order linear differential equation to an $(n-1)$ st order nonlinear differential equation, and so another approach had to be developed.

The coefficients are assumed to lie in a differential field $D$ such that, if $f \in D$ and $f \neq 0$, then $f \sim a x^{b}$ for some complex number $a \neq 0$ and real $b$. In [7], Strodt proved that algebraic closures of such fields exist which retain the asymptotic property. Thus we can assume that $D$ is algebraically closed. This property is necessary in Lemma 8.5 . The results are not possible wihout the field condition on the coefficients.

The technique we develop for $n$th order linear differential equations involves successive changes of variables of the form $y=z \exp \int{ }^{x} N$. By factoring out $\exp \int^{x} N$, the resulting equation is again of the same form. The field condition allows us to choose $N$ so that eventually these changes of variable lead us into the nonexceptional case, so that results from [1] can be applied. We show that there exists a fundamental set of solutions, $p$ of which are of the form $y \sim x^{\alpha}(\log x)^{\beta}$ and $n-p$ of which are of the form $u(x) \exp \int^{x} V$, where $u(x)$ is as above and $V$ is asymptotically equivalent to a root of $H$. The technique is easier to apply than classical formal system expansion.

For the field $D$, the results are essentially the same as the classical result. We emphasize that the coefficients need not be defined in a neighborhood of $\infty$ and we can read asymptotic behavior of the solutions directly from the equation. By Strodt [7, p. 247], the field of functions with representation around $\infty, f(x)=x^{p / q} \sum_{i=0}^{\infty} c_{i} x^{i / q}$ where the $c_{i}^{\text {'s }}$ are complex, $p$ and $q$ are 
integers and $q<-1$, is algebraically closed and in fact also closed under differentiation. In this case, Theorem 5 gives the asymptotic analog to the neighborhood branch solutions of linear ordinary differential equations.

Although the result relies upon the existence theorem of Strodt [7, p. 244] and hence upon Zorn's lemma, in general, the technique applies in constructive fashion. This fact is demonstrated in the examples in $\$ 14$.

2. Concepts from [1], [2], [6] and [7].

2.1 Notation. Let $-\pi<a<b<\pi$. For each nonnegative real valued function $g$ on $(0,(b-a) / 2)$, let $E(g)$ be the union over $\delta \in(0,(b-a) / 2)$ of all sectors, $a+\delta<\arg (x-h(\delta))<b-\delta$ where $h(\delta)=g(\delta) \exp (i(a+$ $b) / 2$ ). The set of all $E(g)$ over all $g$ is denoted $F(a, b)$ and is a filter base which converges to $\infty$ (see $[6, \S 94])$. Each $E(g)$ is simply connected by [6, $\S 93]$. The set $F(a, b)$ is a complex neighborhood system of $\infty$. As in [6, $\$ 8$ and 9], we let $S_{1}$ be the subset of $F(a, b)$ consisting of those members $E$ of $F(a, b)$ such that $|z|>1$ for all $z \in E$. By $\log F(a, b)$ is meant the set $\{\log R$ : $R \in S_{1}$ \}. This set is again a complex neighborhood system of $\infty$. For $W$ analytic in $E(g)$, let $\int W$ stand for a primitive of $W$ in $E(g)$. A statement is said to hold except in finitely many directions (briefly e.f.d.) in $F(a, b)$ if there are finitely many points $r_{1}<r_{2}<\cdots<r_{q}$ in $(a, b)$ such that the statement holds in each of $F\left(a, r_{1}\right), F\left(r_{1}, r_{2}\right), \ldots, F\left(r_{q}, b\right)$ separately (see [1, §6]).

2.2 Notation. The asymptotic relations used are due to Strodt [6, §13]. If $f$ is analytic in some $E(g)$, then $f \rightarrow 0$ in $F(a, b)$ means that for any $\varepsilon>0$, there is a $g_{1}$ such that $|f(x)|<\varepsilon$ for all $x$ in $E\left(g_{1}\right)$. By $f<1$ in $F(a, b)$ is meant that in addition to $f \rightarrow 0$, all functions $\theta_{j}^{k} f \rightarrow 0$, where $\theta_{j}^{k} f=\theta_{j}\left(\theta_{j}^{k-1} f\right)$ with $\theta_{j} f=$ $x \log x \ldots \log _{j-1} x f^{\prime}$ and where $\log _{m} x$ is the iterated $m$-fold logarithm. Then $f_{1} \prec f_{2}, f_{1} \sim f_{2}, f_{1} \approx f_{2}$ and $f_{1} \prec f_{2}$ mean respectively $f_{1} / f_{2} \prec 1, f_{1}-f_{2} \prec f_{2}$, $f_{1} \sim c f_{2}$ for some nonzero constant $c$ and finally either $f_{1}<f_{2}$ or $f_{1} \approx f_{2}$. If $f \sim c$ we write $f(\infty)=c$, while if $f \prec 1$ we write $f(\infty)=0$.

2.3 Definition. By a complex logarithmic monomial $M$ is meant a function of the form

$$
M(x)=K x^{a_{0}}(\log x)^{a_{1}} \ldots\left(\log _{p} x\right)^{a_{p}}
$$

where $K, a_{0}, a_{1}, \ldots, a_{p}$ are complex and $K \neq 0$. We say $M$ has rank $<p$. If the $a_{k}$ 's are real we say that $M$ is a logarithmic monomial.

2.4 RemarK. In [6, \$28], Strodt showed that if $f \prec 1$ then $\theta_{j} f \prec 1$ for all $j$. Additionally if $f \prec x^{a}$ for real $a$, then $f^{\prime} \prec x^{a-1}$ and if $f \prec x^{a}$ for real $a$, then

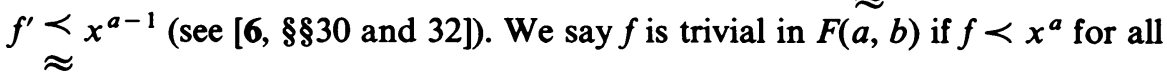
real $a$. In accordance with $[6, \S 23]$, if $f \sim K x^{a_{0}}(\log x)^{a_{1}} \ldots\left(\log _{q} x\right)^{a_{q}}$, then $\delta_{j}(f)$ denotes $a_{j}$ and if $f$ is trivial, then $\delta_{0}(f)=-\infty$. 
2.5 Definition. A logarithmic differential field of rank $q>0$ (briefly an $\mathrm{LDF}_{q}$, [7, p. 247]) over $F(a, b)$ is a field $D$ of functions (each analytic in some $E(g))$ such that $D$ is closed under differentiation, $D$ contains all logarithmic monomials of rank $\leqslant q$ and such that every nonzero element of $D$ is $\sim$ a logarithmic monomial of rank $\leqslant q$.

2.6 Remark. Let $D$ be an $\mathrm{LDF}_{q}$ over $F(a, b)$. By [7, Theorem II, p. 244] there exists an algebraically closed extension field $E$ of $D$ such that $E$ contains all logarithmic monomials of rank $\leqslant q$ and every nonzero element of $E$ is $\sim$ a logarithmic monomial of rank $<q$. Note that $E$ is not necessarily a differential field. Let $F=\{f \in E: f$ is algebraic over $D\}$. The field $F$ is an algebraically closed $\mathrm{LDF}_{q}$ which contains $D$.

2.7 Definition. Let $\Omega$ be the $n$th order linear differential polynomial $\Omega(y)=\sum_{j=0}^{n} f_{j}(x) y^{(j)}$. As in $[1, \S 3], \Omega[j]$ denotes $f_{j}$ and $\Omega[*]=\max \left\{\delta_{0}(\Omega[j])-\right.$ $j: j=0,1, \ldots, n\}$. Let $\alpha$ be a complex number. Let $\Psi(u, v)$ be the transform of $\Omega(y)$ under the change of variables $x=e^{u}$ and $y=v e^{\alpha u}$. Then the differential polynomial $\exp (-\Omega[*, \alpha] u) \Psi(u, v)$ is denoted $[\alpha ; \Omega](v)$, where $\Omega[*, \alpha]$ is $\alpha+\Omega[*]$ (see $[2, \S 7])$. For a nonlinear differential polynomial $\Omega$, when $i^{*}=\left(i_{0}, i_{1}, \ldots, i_{n}\right)$ is an $(n+1)$-tuple of natural numbers, the coefficient of $y^{i_{0}}\left(y^{\prime}\right)^{i_{1}} \ldots\left(y^{(n)}\right)^{i_{n}}$ is denoted $\Omega\left[i^{*}\right]$. Let $d\left(i^{*}\right)=i_{0}+i_{1}$ $+\ldots+i_{n}$ and $w\left(i^{*}\right)=i_{1}+2 i_{2}+\ldots+n i_{n}$. Then $\Omega[*, \alpha]$ means the maximum over $i^{*}$ of the quantity $\alpha d\left(i^{*}\right)+\delta_{0}\left(\Omega\left[i^{*}\right]\right)-w\left(i^{*}\right)$.

2.8 Definition. We say that $M$ is a complex critical monomial of $\Omega$ if $\Omega$ is unstable at $M$; that is, there exists a function $h \sim M$ such that $\Omega(h)$ is not $\sim \Omega(M)$. Then we write $M \in \operatorname{crit}^{*}(\Omega)$.

\subsection{Definition. If}

$$
M \sim c x^{-1}(\log x)^{-1} \ldots\left(\log _{k-1} x\right)^{-1}\left(\log _{k} x\right)^{-1+t}\left(\log _{k+1} x\right)^{c_{1}} \ldots\left(\log _{k+r} x\right)^{c_{r}}
$$

with $t>0$, then by $I F(M)$ is meant the function $\cos \left(\delta_{0 k} t \gamma+\arg c\right)$ for $-\pi<\gamma<\pi$, where $\delta_{0 k}$ is the Kronecker delta. This is the indicial function (see $[6, \S 82])$.

\section{Uniform hypotheses.}

3.1 Notation. The operator $\Omega$ is an $n$th order linear differential polynomial of the form $\Omega(y)=\sum_{k=0}^{n} B_{k}(x) \theta^{k} y$, where $\theta y=x y^{\prime}$ and $\theta^{k} y=\theta\left(\theta^{k-1} y\right)$. The coefficients $B_{k}(x)$ are assumed to lie in an algebraically closed LDFo, say $D$, over $F(a, b)$. Assume that $B_{n}(x)$ is not identically zero. By dividing by the highest power of $x$ which is $\sim$ a coefficient $B_{k}(x)$, we may assume that for each $k, B_{k}(x) \prec 1$ and for some $k, B_{k}(x) \approx 1$. We write $B_{k}(x)=x^{\alpha_{k}}\left[a_{k}+\right.$ $F_{k}(x)$ ] where $\delta_{0}\left(F_{k}\right)<0$ and $\alpha_{k} \leqslant 0$ for all $k$. Let $p$ be the nonnegative integer such that $B_{p}(x) \approx 1$ and for some $\delta>0, B_{k}(x) \prec x^{-\delta}$ for $k>p$. This $p$ is called the critical degree. 
3.2 Definition. The critical equation of $\Omega$ is the equation defined in $[5, \S 4]$ as $F(a)=\sum_{j=0}^{n} B_{j}(\infty) a^{j}=0$. It is clear from $\$ 3.1$ that $F(a)$ is a polynomial of degree $p$.

\section{4.}

MAIN TheOREM OF [1]. Assume §3. Let $q=\min \left\{j: B_{j} \approx 1\right\}$. By dividing $\Omega$ by $B_{q}(\infty)$ we may assume that $B_{q}(x) \sim 1$. If $q>0,0$ is a root of $F$ of multiplicity $q$; otherwise, 0 is not a root of $F$. Let $r_{1}, r_{2}, \ldots, r_{s}$ be the distinct nonzero roots of $F$, each $r_{j}$ having multiplicity $m_{j}$. Define $M_{1}, M_{2}, \ldots, M_{p}$ as follows: $M_{j}=(\log x)^{j-1}$ if $1<j<q ; M_{q+j}=x^{r_{1}}(\log x)^{j-1}$ if $1<j \leqslant m_{1}$ and in general, $M_{q+m_{1}+m_{2}+\cdots+m_{k}+j}=x^{r_{k+1}}(\log x)^{j-1}$ for $1<k<s-1$ and $1 \leqslant$ $j<m_{k+1}$. Then the equation $\Omega(y)=0$ possesses e.f.d. in $F(a, b)$, a linearly independent set of solutions $\left\{g_{1}, g_{2}, \ldots, g_{p}\right\}$ with $g_{j} \sim M_{j}$ for $j=1,2, \ldots, p$.

Proof. The proof is given in $[1, \S \S 5,7$ and 10$]$.

\section{5.}

MaIn Theorem. Assume §3. Let $H(w)=\sum_{k=0}^{n} x^{k} B_{k}(x) w^{k}$ and let $A$ be the sequence of critical monomials $N$ of $H$ with $\delta_{0}(N)>-1$ including multiplicity. Then:

(1) $A$ has precisely $n-p$ elements including multiplicity, say $N_{1}, N_{2}, \ldots, N_{n-p}$, and e.f.d. in $F(a, b), \Omega(y)=0$ possesses a solution of the form $y_{j} \sim u_{j} \exp \int V_{j}$ for $j=1,2, \ldots, n-p$ where $V_{j} \sim N_{j}$ and $u_{j} \sim$ $x^{r_{j}}(\log x)^{k_{j}}$ for some complex number $r_{j}$ and nonnegative integer $k_{j}$.

(2) For $j=1,2, \ldots, n-p, N_{j}$ is of the form $c_{j} x^{-1+d_{j}}$ with $d_{j}>0$ and, if $\left(a_{1}, b_{1}\right)$ is a subinterval of $(a, b)$ on which $\operatorname{IF}\left(N_{j}\right)>0$ (respectively $\left.\operatorname{IF}\left(N_{j}\right)<0\right)$ and is such that $y_{j}$ exists on $F\left(a_{1}, b_{1}\right)$, then for all real $\alpha, y_{j} \succ x^{\alpha}$ (respectively $\left.y_{j} \prec x^{\alpha}\right)$ in $F\left(a_{1}, b_{1}\right)$.

(3) There exist $p$ distinct functions $M_{1}, M_{2}, \ldots, M_{p}$ where $M_{k}=x^{\gamma_{k}}(\log x)^{\beta_{k}}$ for some complex $\gamma_{k}$ and nonnegative integer $\beta_{k}$ such that, e.f.d. in $F(a, b)$, the equation $\Omega(y)=0$ possesses a fundamental set of solutions $\left\{g_{1}, g_{2}, \ldots, g_{p}, y_{1}\right.$, $\left.y_{2}, \ldots, y_{n-p}\right\}$ where the $y_{j}^{\prime}$ 's are as in (1) and $g_{k} \sim M_{k}$ for $k=1,2, \ldots, p$.

Proof. The theorem summarizes a sequence of lemmas and the proof is given in $\$ 13$.

\section{Lemmas on critical monomials.}

6.1 LemMA. Assume \$3. Let $H(w)=\sum_{k=0}^{n} x^{k} B_{k}(x) w^{k}$ and $H_{p}(w)=$ $\sum_{k=p}^{n} x^{k} B_{k}(x) w^{k-p}$. Then the critical monomials of $H_{p}$ are precisely those critical monomials $M$ of $H$ with $M>x^{-1}$. This is the sequence $A$ of Theorem 5. Hence $A$ has precisely $n-p$ monomials. Also, if $N \in A$, then $N=c x^{\alpha}$ for some complex number $c$ and real $\alpha>-1$. 
Proof. Since $H_{p}(w)$ has coefficients in $D$, it follows from [2, §8] and [6, \$54] that, if $\alpha$ is any real number, $\left[\alpha ; H_{p}\right](v)$ is the sum of a polynomial having constant coefficients with one having trivial coefficients. That is, $[\alpha$; $\left.H_{p}\right](v)=C(v)+R(v)$ where $C(v)$ is a polynomial with constant coefficients and $R(v)$ is trivial in $\log F(a, b)$. A real number $\alpha$ is called admissible for $H$ if $H^{(r)}[*, \alpha]=H[*, \alpha]=H^{(q)}[*, \alpha]$ holds for at least two distinct $r$ and $q$. Here by $H^{(q)}$ we mean the homogeneous part of degree $q$. It follows from $[2, \S 26]$ that any critical monomial of $H_{p}$ must be of the form $c x^{\alpha}$ for some nonzero $c$ and admissible $\alpha$, since $\left[\alpha ; H_{p}\right](v)$ has zero and only zero as an admissible value.

Let $\alpha$ be admissible and let $J=\left\{k: \alpha_{k}+k+(k-p) \alpha=H[*, \alpha], p<k\right.$ $<n\}$. Then, $\left[\alpha ; H_{p}\right](v)=\sum_{k \in J} a_{k} v^{k-p}+T(v)$, where $T(v)$ is trivial in $\log F(a, b)$. Since $\alpha$ is admissible, $\alpha_{k}+k+(k-p) \alpha \geqslant p+\alpha_{p}$ for some $k>p$, and since $\alpha_{p}=0$ and $\delta_{0}\left(B_{k}\right)=\alpha_{k}<0$ for $k>p$, we must have $\alpha>-1$.

Now consider $H$. Let $\alpha>-1$. For each $j<p, \alpha_{j}+j+j \alpha \leqslant \alpha_{p}+j+j \alpha$ $<\alpha_{p}+p+p \alpha$. Since $H[*, \alpha]=\max \left\{\alpha_{k}+k+k \alpha: 0 \leqslant k \leqslant n\right\}$, then for $j<p, \alpha_{j}+j(\alpha+1)<H[*, \alpha]$. Clearly, $H[*, \alpha]=H_{p}[*, \alpha]+p \alpha$. So, $[\alpha$; $H](v)=\sum_{k \in J} a_{k} v^{k}+T_{1}(v)$, where $T_{1}(v)$ is trivial in $\log F(a, b)$. It follows that $[\alpha ; H](v)=v^{p}\left[\alpha ; H_{p}\right](v)-v^{p} T(v)+T_{1}(v)$. The nontrivial portion of $[\alpha ; H](v)$ is precisely the nontrivial portion of $v^{p}\left[\alpha ; H_{p}\right](v)$. So for admissible $\alpha>-1$, the critical monomials are identical. This completely characterizes

$A$. Since the terms of degree $n-p$ and 0 in $H_{p}$ are nontrivial, it follows from $[2, \S 29(\mathrm{~b})]$ that $A$ contains exactly $n-p$ elements. This completes the proof.

6.2 LeMma. Assume \$3. Let $A=\left\{N_{1}, N_{2}, \ldots, N_{n-p}\right\}$ where $N_{1} \approx \cdots \approx$ $N_{s_{1}} \prec N_{s_{1}+1} \approx \cdots \approx N_{s_{2}} \prec \cdots \prec N_{s_{m-1}+1} \approx \cdots \approx N_{s_{m}}=N_{n-p}$. Let $s_{0}=$ 0. Let $\delta_{0}\left(N_{s_{j}}\right)=T_{j}$ for $j=1,2, \ldots$, m. Let $\left[T_{j} ; H\right](v)=f_{j}(v)+R_{j}(v)$ where $R_{j}(v)$ is trivial in $\log F(a, b)$ and $f_{j}(v)$ is a polynomial with constant coefficients for each $j$. Then for $j=1,2, \ldots, m$,

(1) $f_{j}(v)=v^{p+s_{j-1}} g_{j}(v)$ with $g_{j}(0) \neq 0$.

If $N_{1}=N_{2}=\cdots=N_{r} \prec N_{k}$ for $k>r$, then

(2) $B_{k}(x) \sim\left(\begin{array}{c}r \\ k-p\end{array}\right) B_{r+p}(x)\left(-x N_{1}\right)^{p+r-k}$ for $k=p, p+1, \ldots, p+r$.

Proof. Let $N_{1} \sim a x^{T}$. Clearly, $T$ is the minimum admissible value which is $>-1$. Set $J=\left\{k: \alpha_{k}+k+k T=H[*, T]\right\}$. Let $\beta=\min \left\{-1+\left(\alpha_{p}-\right.\right.$ $\left.\left.\alpha_{k}\right) /(k-p): k>p\right\}$ and let $t$ be the maximum $k$ for which equality holds. Since $\alpha_{k}<0$ for $k>p$, then $\beta>-1$. Note that $\alpha_{p}=0, \alpha_{t}+t+t \beta=p+$ $p \beta$ and $\alpha_{k}+k+k \beta<p+p \beta$ for all $k$. Thus, $\beta$ is admissible for $H$. Recall that if $a_{k}=0$, then $\alpha_{k}=-\infty$.

Let $-1<\alpha<\beta$. Then, $\alpha_{k}+k+k \alpha \leqslant k+k \alpha<p+p \alpha$ for $k<p$ and $\alpha_{k}+k+k \alpha=\alpha_{k}+k+k \beta+k(\alpha-\beta)<p+p \beta+p(\alpha-\beta)=p+p \alpha$ for $k>p$. Hence, $\alpha$ is not admissible and so $\beta=T$. It then follows that 
$J \subset\{p, p+1, \ldots, t\}$. So, $[T ; H](v)=\Sigma_{k \in J} a_{k} v^{k}+T(v)$ where $T(v)$ is trivial in $\log F(a, b)$. Now since $H$ has $s_{1}-s_{0}$ monomials $N$ in $A$ with $\delta_{0}(N)=T$, then $t=p+s_{1}$ and $f_{1}(v)=\Sigma_{k \in J} a_{k} v^{k}=v^{p} g_{1}(v)$ with $g_{1}(0) \neq 0$. Assume that part (1) holds for $j=1,2, \ldots, q$ with $q<m$. Then

$$
T_{q+1}=\min \left\{-1+\left(\alpha_{p+s_{q}}-\alpha_{k}\right) /\left(k-p-s_{q}\right): k>p+s_{q}\right\} .
$$

Set $J_{q+1}=\left\{k: \alpha_{k}+k+k T_{q+1}=H\left[*, T_{q+1}\right]\right\}$. Therefore, $p+s_{q}$ is in $J_{q+1}$ and $J_{q+1} \subset\left\{p+s_{q}, \ldots, n\right\}$. Hence, $f_{q+1}(v)=\Sigma_{k \in J_{q+1}} a_{k} v^{k}=v^{p+s_{q}} g_{q+1}(v)$, where $g_{q+1}(0) \neq 0$, since $f_{q+1}(v)$ has a $p+s_{q}$-fold root at zero.

If $N_{1}$ has multiplicity $r$ and $N_{r} \prec N_{k}$ for $k>r$, then $a_{r+p}(v+a)^{r}=$ $\Sigma_{k \in J} a_{k} v^{k-p}$. Clearly, $J$ is precisely $\{p, p+1, \ldots, p+r\}$ and $a_{k}=$ $a_{r+p}\left(\begin{array}{c}r \\ k-p\end{array}\right) a^{r+p-k}$. Also we must have $\alpha_{k}+k+k T=p+p T$ for $k \in J$ so that $\alpha_{k}=\alpha_{r+p}+(p+r-k)(T+1)$. Thus

$$
B_{k}(x) \sim a_{k} x^{\alpha_{k}} \sim B_{r+p}(x)\left(\begin{array}{c}
r \\
k-p
\end{array}\right)\left(-x N_{1}\right)^{r+p-k} \quad \text { for } k \in J .
$$

This completes the proof.

7. The transform. Let $W \in D$ (the $\mathrm{LDF}_{0}$ containing the coefficients of $\Omega$ ) with $W$ not identically zero. Set $s=\exp \int W$ and $y=s z$. We write $\theta^{k}(s)=$ $s(x W)^{k}\left(1+E_{k}\right)$. Clearly $\theta^{0} s=s$ and $\theta s=x W s$. So, $E_{0}=E_{1}=0$. The equation becomes $\Omega(s z)=s \Lambda(z)=0$, where $\Lambda(z)=\sum_{j=0}^{n} B_{j}[W] \theta^{j} z$, with $B_{j}[W]=$ $\sum_{k=j}^{n}\left(\begin{array}{l}k \\ j\end{array}\right) B_{k}(x)(x W)^{k-j}\left(1+E_{k-j}\right)$. Simple induction shows that $E_{k}$ and $B_{k}[W]$ are in $D$ for all $k$. Also, since $W$ is in $D, W \approx x^{a}$ for some $a$. Hence by $\S 2.4$, $W^{\prime} \prec x^{a-1} \approx x^{-1} W$. Then $W^{(n)} \prec x^{-n} W$. A straightforward computation using the above fact shows that for $W>x^{-1}$,

$$
\theta^{m} s=s\left[(x W)^{m}+\left(\begin{array}{c}
m \\
2
\end{array}\right)(x W)^{m-1}+\left(\begin{array}{c}
m \\
2
\end{array}\right) x^{m} W^{m-2} W^{\prime}+T_{m}\right] \text { for } m \geqslant 2 \text {, }
$$

where $T_{m} \in D$ and $T_{m} \precsim(x W)^{m-2} \prec(x W)^{m-1}$. It then follows readily that $E_{m} \prec 1$ for $m \geqslant 2$. However, $E_{m} \in D$ and thus $\delta_{0}\left(E_{m}\right)<0$.

Assume that $W>x^{-1}$. Define $G_{m}=(x W)^{m} E_{m}$. Thus, $G_{0}=G_{1}=0$. Set

$$
D_{j}[W]=\sum_{k=j}^{n}\left(\begin{array}{l}
k \\
j
\end{array}\right) B_{k}(x)(x W)^{k-j} \quad \text { and } \quad S_{j}[W]=\sum_{k=j}^{n}\left(\begin{array}{l}
k \\
j
\end{array}\right) B_{k}(x) G_{k-j}
$$

Therefore, $B_{j}[W]=D_{j}[w]+S_{j}[W]$. Note that since $E_{m} \in D$, then $G_{m} \in D$ and hence, $D_{j}[W]$ and $S_{j}[W]$ are in $D$.

\section{Estimates.}

8.1 Definition. If $f \sim k x^{a_{0}}(\log x)^{a_{1}} \ldots\left(\log _{q} x\right)^{a_{q}}$ then $] f[$ means the monomial $x^{a_{0}}(\log x)^{a_{1}} \ldots\left(\log _{q} x\right)^{a_{q}}$ and is called the gauge of $f$.

8.2 Uniform hypotheses. Let $H(w)=\sum_{k=0}^{n} x^{k} B_{k}(x) w^{k}$. Let $a x^{T} \in A$ and let $N \in D$ with $N \sim a x^{T}$. Thus $N=a x^{T}(1+E)$ where $E \prec 1$ and in fact $\delta_{0}(E)<0$. Let $K(w)=\sum_{k=0}^{n} x^{k} B_{k}[N] w^{k}$. Assume that $a x^{T}$ is an $r$ th repeated critical monomial of $H$. Set $m=\max \{] x^{k} B_{k}(x) N^{k}[: 0 \leqslant k \leqslant n\}$. 
8.3 Lemma. Assume §8.2. Let $[T ; H](v)=f(v)+T(v)$ with $T(v)$ trivial in $\log F(a, b)$. Then,

(1) $\left.B_{r}[N] \sim\right] m N^{-r} x^{-r}\left[f^{(r)}(a) / r !\right.$,

(2) $\left.B_{j}[N] \prec\right] m N^{-j} x^{-j}[$ for $j<r$, and

(3) $B_{j}[N] \underset{\approx}{\prec} m N^{-j} x^{-j}\left[\prec B_{r}[N]\right.$ for $j>r$.

Proof. Since $H[*, T]=\max \left\{\alpha_{k}+k+k T\right\}$, then $\delta_{0}(m)=H[*, T]$. Let $J=\left\{k: a_{k} \neq 0\right\}$ and recall that $a_{k}=0$ implies $B_{k}(x) \equiv 0$. Let $J^{\prime}=J \cap\{j, j$ $+1, \ldots, n\}$. By the assumptions in $\$ \S 3.1$ and 8.2 we may write

$$
B_{j}[N]=\sum_{k \in J^{\prime}}\left(\begin{array}{l}
k \\
j
\end{array}\right) x^{\alpha_{k}+(k-j)(T+1)} a^{k-j} a_{k}\left(1+F_{k} / a_{k}\right)(1+E)^{k-j}\left(1+E_{k-j}\right) .
$$

Let $I=\left\{k: \alpha_{k}+k+k T=H[*, T]\right\}$. Clearly $I \subset J$. Let $I^{\prime}=I \cap\{j, j+$ $1, \ldots, n\}$. Since $F_{k} \prec 1, E \prec 1$ and $E_{k-j} \prec 1$, we have

$$
B_{j}[N]=x^{-j(T+1)} \sum_{k \in J^{\prime}}\left(\begin{array}{l}
k \\
j
\end{array}\right) x^{\alpha_{k}+k(T+1)} a^{k-j} a_{k}\left(1+G_{k j}\right)
$$

where $\delta_{0}\left(G_{k j}\right)<0$. Hence,

$$
B_{j}[N]=x^{H[*, T]-j(T+1)}\left(\sum_{k \in I^{\prime}}\left(\begin{array}{l}
k \\
j
\end{array}\right) a^{k-j} a_{k}+H_{j}\right)
$$

where $\delta_{0}\left(H_{j}\right)<0$. By assumption and the definition of $H[*, T],[T ; H](v)=$ $f(v)+T(v)$, where $T(v)$ is trivial in $\log F(a, b)$. It follows from the definition of $I$ that $f(v)=\sum_{k \in I} a_{k} v^{k}$. Differentiate to get $f^{(j)}(a) / j !=\sum_{k \in I^{\prime}}\left(\begin{array}{l}k \\ j\end{array}\right) a_{k} a^{k-j}$. Thus,

$$
B_{j}[N]=x^{H[*, T]-j(T+1)}\left(f^{(j)}(a) / j !+H_{j}\right) .
$$

Now $a x^{T}$ is an $r$ th repeated critical monomial of $H$. Therefore, $f^{(j)}(a)=0$ for $j<r$ and $f^{(r)}(a) \neq 0$. Since $\left.x^{H[*, T]-j(T+1)}=\right] m x^{-j} N^{-j}[$, parts (1) and (2) follow. Also $x N \prec 1$, so for $j>r$ we have ] $m x^{-j} N^{-j}[\prec] m x^{-r} N^{-r}[$. Part (3) then follows. This completes the proof.

8.4 Lемма. Assume §8.2. Then $S_{n-1}[N]=S_{n}[N]=0$ and $\left.S_{j}[N] \underset{\approx}{\approx}\right] m x^{-j-1} N^{-j-1}[$ for $j<n-2$.

Proof. Since $G_{0}=G_{1}=0$, the first part is clear. From $\$ 7$ we may write $S_{j}[N]=\sum_{k=j+2}^{n}\left(\begin{array}{l}k \\ j\end{array}\right) B_{k}(x) G_{k-j}$ with $G_{k}=\left(\begin{array}{l}k \\ 2\end{array}\right)(x N)^{k-1}+\left(\begin{array}{l}k \\ 2\end{array}\right) x^{2}(x N)^{k-2} N^{\prime}+T_{k}$ and with $T_{k}<(x N)^{k-1}$.

If $T=0$, then $N=a+E$ with $E \prec 1$. Thus, $N^{\prime} \prec x^{-1}$ and $G_{k} \sim$ $\left(\begin{array}{l}k \\ 2\end{array}\right)(x N)^{k-1}$. If $T \neq 0, N=a x^{T}(1+E)$. Then $N^{\prime}=x^{-1} N\left[T+x E^{\prime} /(1+\right.$ $E)]$. So, $G_{k}=(T+1)\left(\begin{array}{c}k \\ 2\end{array}\right)(x N)^{k-1}+Q_{k}$, where $Q_{k} \prec(x N)^{k-1}$. Thus, $G_{k}$ $\approx](x N)^{k-1}\left[\right.$. Since $G_{k}$ and $S_{j}[N]$ lie in $D$, it is sufficient to check $\delta_{0}\left(S_{j}[N]\right)$. 
We have

$$
\begin{aligned}
\delta_{0}\left(S_{J}[N]\right) & \leqslant \max \left\{\delta_{0}\left(B_{k}(x) G_{k-j}\right): k \geqslant j+2\right\} \\
& \leqslant \max \left\{\alpha_{k}+(k-j-1)(T+1): k \geqslant j+2\right\} \\
& \leqslant H[*, T]-(j+1)(T+1) .
\end{aligned}
$$

Therefore, $\delta_{0}\left(S_{j}[N]\right) \leqslant \delta_{0}(m)-(j+1)(T+1)$ and hence, $S_{j}[N] \underset{\approx}{\prec} m(x N)^{-j-1}[$ for $j \leqslant n-2$. This completes the proof.

8.5 Lemma. Assume §8.2. If $D_{r-1}[N]>m x^{-r} N^{-r}$, then there exists a function $M \in D$ such that $x^{-1} \prec M \prec N$ and $D_{r-1}[N+M]=0$.

Proof. By $\$ 8.2$ and the assumption, $m x^{-r} N^{-r} \prec$ $D_{r-1}[N] \prec m x^{-r+1} N^{-r+1}$. Since $N>x^{-1}$, it follows from Lemma 8.4 that $S_{r-1}[N] \stackrel{\approx}{\prec} m x^{-r} N^{-r} \prec m x^{-r+1} N^{-r+1}$. Also, by Lemma 8.3, $B_{r-1}[N] \prec$ $m x^{-r+1} \widetilde{\widetilde{N}}^{-r+1}$ and therefore $D_{r-1} \prec m x^{-r+1} N^{-r+1}$. Now $D_{r-1}[N]$ is nonzero by hypothesis, so $D_{r-1}[N] \sim m x^{-\delta}$ where $0<r(T+1)-\delta<T+1$. Since $S_{r}[N] \prec m x^{-r} N^{-r}$ by Lemma 8.4 and $B_{r}[N] \approx m x^{-r} N^{-r}$ by Lemma 8.3, then $D_{r}[N] \approx m x^{-r} N^{-r}$. Thus, $D_{r}[N]$ is nonzero.

Let $A(v)=D_{j}[N+v]$. By Taylor's Theorem, $A(v)=\sum_{p-0}^{n-j} A^{(p)}(0) v^{p} / p !$. Hence, with $p=m-j$,

$$
A(v)=\sum_{m=j}^{n}\left(\begin{array}{c}
m \\
j
\end{array}\right)\left[\sum_{k=m}^{n}\left(\begin{array}{l}
k \\
m
\end{array}\right) B_{k}(x) x^{k-m} N^{k-m}\right] x^{m-j} v^{m-j} .
$$

So, $\quad D_{j}[N+v]=\sum_{k=j}^{n}\left(\begin{array}{l}k \\ j\end{array}\right) D_{k}[N] x^{k-j} v^{k-j}$. Set $G(v)=D_{r-1}[N+v]$. Since $-D_{r-1}[N] / r x D_{r}[N]$ is in $D$, it is $\sim$ a logarithmic monomial, say $B$. We have shown that $B \approx x^{r(T+1)-\delta-1}$ and $x^{-1} \prec B \prec N$.

Let $\gamma_{j}=\delta_{0}\left(D_{j}[N]\right)$ and $\alpha=\delta_{0}(B)$. Now $\gamma_{k} \leqslant \delta_{0}(m)-k(T+1)$ for any $k$ and $\gamma_{r}=\delta_{0}(m)-r(T+1)$. Hence, $\gamma_{r}-\gamma_{k}>(k-r)(T+1)$. For $k>r$, $\gamma_{k}+(k-r+1)(\alpha+1) \geqslant \gamma_{r}+\alpha+1$ is false. If not, $(k-r)(\alpha+1)>\gamma_{r}-$ $\gamma_{k}$ which implies that $\alpha \geqslant T$. However, $\alpha<T$ and so $\gamma_{k}+(k-r+1)(\alpha+$ 1) $\left\langle\gamma_{r}+\alpha+1\right.$ for $k>r$. By the choice of $\alpha, \gamma_{r}+\alpha+1=\gamma_{r-1}$ and so $G[*, \alpha]=\gamma_{r-1}$. Then $\alpha$ is admissible for $G$. Clearly $B$ is in crit* $(G)$ and is simple.

Since $D$ is algebraically closed, $G(v)$ factors over $D$ into linear factors. There then exists a function $M \in D$ with $G(M)=0$ and $M \sim B$. The remainder of the proof follows by the definition of $G, B$ and the fact that $x^{-1} \prec B \prec N$. This concludes the proof.

\section{9.}

Lemma. Assume \$8.2. Let $B$ be the sequence of monomials $M \in A$ such that $M \approx N$ but not $M \sim N$. For each $M \in B, M-a x^{T}$ is in crit* $(K)$ and has the 
same multiplicity as the multiplicity of $M$ in $A$. If $A$ has $t$ monomials $L \prec N$, then $K$ has a critical monomial $-a x^{T}$ of multiplicity $p+t$ where $p$ is the critical degree. Finally, if $M \in A$ and $M>N$, then $M$ is in crit*(K) with the same multiplicity as the multiplicity of $M$ in $A$.

Proof. Let $I=\left\{k: \alpha_{k}+k(T+1)=H[*, t]\right\}$ and $J=\left\{j: \delta_{0}\left(B_{j}[N]\right)+\right.$ $j(T+1)=K[*, T]\}$. Then, $[T ; H](v)=f(v)+T(v)$, where $f(v)=\Sigma_{k \in I} a_{k} v^{k}$ and where $T(v)$ is trivial in $\log F(a, b)$. By definition,

$$
K[*, T]=\max \left\{\delta_{0}\left(B_{j}[N]\right)+j(T+1): 0 \leqslant j \leqslant n\right\} .
$$

Since $\delta_{0}\left(B_{j}[N]\right) \leqslant \max \left\{\alpha_{k}+k(T+1)-j(T+1): j \leqslant k \leqslant n\right\} \leqslant H[*, T]-$ $j(T+1)$, then $K[*, T] \leqslant H[*, T]$. By Lemma 8.3, $B_{r}[N]$ $\sim] m x^{-r} N^{-r}\left[f^{(r)}(a) / r\right.$ ! with $f^{(r)}(a) \neq 0$. Thus, $\delta_{0}\left(B_{r}[N]\right)=\delta_{0}(m)-r(T+1)$ $=H[*, T]-r(T+1)$. Therefore, $H[*, T] \leqslant K[*, T]$. Necessarily the coefficient of $v^{r}$ in $[T ; K](v)$ is nontrivial and so $K[*, T]=H[*, T]$.

For $j \notin J$, the coefficient of $v^{j}$ is trivial. Also with $I^{\prime}=I \cap\{j, j+$ $1, \ldots, n\}, f^{(j)}(a) / j !=\sum_{k \in I}\left({ }_{j}^{k}\right) a_{k} v^{k-j}$. Hence, $[T ; K](v)=$ $\sum_{j \in J} v^{j} f^{(j)}(a) / j !+T_{1}(v)$ where $T_{1}(v)$ is trivial in $\log F(a, b)$. By [2, §8], if $f^{(j)}(a)=0$, then the coefficient of $v^{j}$ is trivial and $f^{(j)}(a)$ is nonzero if and only if $j \in J$. Thus,

$$
f(a+v)=\sum_{k \in J} v^{k} f^{(k)}(a) / k !
$$

Therefore, $[T ; K](v)=f(a+v)+T_{1}(v)$.

Now $K$ (respectively $H$ ) has critical monomial corresponding to admissible value $T$ where and only where $f(a+v)$ (respectively $f(v)$ ) has its nontrivial zeros with the corresponding multiplicity. The first part of the lemma follows. By Lemma 6.2, part (1), $f(v)=v^{p+t} g(v)$ with $g(0) \neq 0$. Hence, $f(a+v)$ has a $p+t$-fold root at $-a$. This proves the second part of the lemma.

Let $\alpha>T$. Define $I=\left\{k: a_{k} \neq 0\right\}$ and $J=\left\{k: \alpha_{k}+k(\alpha+1)=\right.$ $H[*, \alpha]\}$. Clearly $J \subset I$. For $j \in J$ and $k>j$,

$$
\begin{aligned}
\alpha_{k}+(k-j)(T+1) & =\alpha_{k}+k(\alpha+1)-j(T+1)+k(T-\alpha) \\
& \leqslant \alpha_{j}+j(\alpha+1)-j(T+1)+k(T-\alpha) \\
& \leqslant \alpha_{j}+(k-j)(T-\alpha)<\alpha_{j} .
\end{aligned}
$$

Hence, $B_{j}[N] \sim B_{j}(x)$. For $j \notin J, \alpha_{j}+j(\alpha+1)<H[*, \alpha]$ and for $k>j$,

$$
\begin{aligned}
\alpha_{k}+(k-j)(T+1)+j(\alpha+1) & =\alpha_{k}+k(\alpha+1)+(k-j)(T-\alpha) \\
& <H[*, \alpha] .
\end{aligned}
$$

Since $J$ is not empty, $K[*, \alpha] \nless H[*, \alpha]$. Therefore, $K[*, \alpha]=H[*, \alpha]$ and equality occurs at each term with $j \in J$. Then we must have $[\alpha ; K](v)=[\alpha$; $H](v)+T(v)$ where $T(v)$ is trivial in $\log F(a, b)$. The conclusion of the last part of the lemma follows. This completes the proof. 


\section{Fundamental reduction lemmas.}

10.1 Definition. Let $F(y)=\sum_{i=0}^{n} a_{i} y^{i}$ be a polynomial with coefficients in $D$. Let $M^{*}$ be the set of monomials in $D$. Then $F$ is called stable at $p \in M^{*}$ if $F(q) \sim F(p)$ for all $q \in D$ with $q \sim p$. The instability multiplicity for $F$ (briefly inst $(p, F))$ is equal to the $\min \left\{s: F^{(s)}\right.$ is stable at $\left.p\right\}$ (see [7, p. 231]).

10.2 Definition. Let $x^{-1} \prec N \in D$. Let $H(w)=\sum_{k=0}^{n} x^{k} B_{k}(x) w^{k}, K(w)=$ $\sum_{k=0}^{n} x^{k} B_{k}[N] w^{k}$ and $\Lambda(z)=\sum_{k=0}^{n} B_{k}[N] \theta^{k} z$. Then $N$ has transform type $(p, q)$ with respect to $H$ (briefly $\operatorname{trt}(N, H)=(p, q))$ if $\Lambda$ has critical degree $p$ and if $q=\min \left\{\operatorname{inst}(M, K): M \in \operatorname{crit}^{*}(K)\right.$ and $\left.x^{-1} \prec M \prec N\right\}$. Take $q=0$ if $\left\{M: M \in \operatorname{crit}^{*}(K)\right.$ and $\left.x^{-1} \prec M \prec N\right\}$ is empty.

10.3 Lemma. Assume §8.2. Let $\Lambda$ be as in $\$ 10.2$. Assume that $\operatorname{trt}(N, H)=$ $(h, q)$. Then $K$ has precisely $r-h$ critical monomials $M$ with $x^{-1} \prec M<N$ counting multiplicity. Also, $h+q \leqslant r$ so that either $h \geqslant 0$ and $q<r$ or $h=0$ and $q=r$.

Proof. Let $\Omega$ have critical degree $p$. By Lemma 6.1, $H$ has $n-p$ critical monomials $Q$ with $Q \prec x^{-1}$ counting multiplicity. Let $B$ be the sequence of these monomials. Let $t$ be the number of terms $M \in B$ with $x^{-1} \prec M \prec N$, $u$ be the number of terms $M \in B$ with $M \approx N$ but not $M \sim N$, and $v$ be the number of terms $M \in B$ with $M>N$. Since $a x^{T} \sim N$ is an $r$ th repeated critical monomial of $H, n-p=t+u+v+r$. By hypothesis, $\Lambda$ has critical degree $h$. Let $C$ be the sequence of monomials $L \in \operatorname{crit}^{*}(K)$ such that $x^{-1} \prec L$ counting multiplicity. By applying Lemma 6.1 to $K, C$ has exactly $n-h$ terms. By Lemma $9,-a x^{T}$ is in $\operatorname{crit}^{*}(K)$ with multiplicity $p+t$, and $K$ has precisely $u$ critical monomials $M$ with $M \approx N$ but not $M \sim-N$. Also from Lemma 9, $K$ has exactly $v$ critical monomials $M$ with $M>N$. Let $s$ be the number of terms $M \in C$ with $x^{-1} \prec M \prec N$. We may conclude that $s=n-h-p-t-u-v$. Therefore, $s=r-h$. Hence, $K$ has $r-h$ critical monomials with $x^{-1} \prec M \prec N$. This proves the first part of the lemma.

Clearly $q \leqslant s$ by definition. Thus, $h+q \leqslant h+s=r$. Since $h$ and $q$ are nonnegative integers, the second part of the lemma follows. Note that if $q=0$, then $s=0$ and necessarily, $h=r$. This completes the proof.

10.4 Definition. Let $p, q, r$, and $s$ be nonnegative integers. We say that $(p, q)<(s, r)$ if $q<r$.

10.5 Lemma. Assume §8.2. Let $\operatorname{trt}(N, H)=(0, r)$. Then there exists a function $L \in D$ satisfying: (a) $L \sim N$ and (b) $\operatorname{trt}(L, H)=(h, q)<(0, r)$. Additionally, with $M=L-N$, (c) $x^{-1} \prec M \prec N$.

Proof. We first find a function $M \in D$ satisfying (c). Then we will show that $L=N+M$ satisfies (a) and (b). We assume that $\operatorname{trt}(N, H)=(0, r)$. Thus since $r>1$, there exists a monomial $Q \in \operatorname{crit}^{*}(K)$ with inst $(Q, K)=r$ and with $x^{-1}<Q<N$. 
We now assert that $D_{r-1}[N] \succ m x^{-r} N^{-r}$. If not, then

$$
D_{r-1}[N] \underset{\approx}{\prec} x^{-r} N^{-r}
$$

since both sides lie in $D$. This leads to a contradiction as follows. By Lemma 8.4,

$$
S_{r-1}[N] \underset{2}{\prec} m x^{-r} N^{-r} .
$$

Hence, $B_{r-1}[N] \prec m x^{-r} N^{-r}$. Let $\Lambda(z)=\sum_{k=0}^{n} B_{k}[N] \theta^{k} z$. Then $\Lambda$ has critical degree 0 . Let $B$ be the sequence of monomials $M \in \operatorname{crit}^{*}(K)$ with $x^{-1} \prec M$ $\prec N$. By Lemma $10.3, B$ has $r$ terms. Now inst $(Q, K)=r$ and $Q \in B$, so $Q$ is the only member of $\operatorname{crit}^{*}(K)$ which satisfies $x^{-1} \prec Q \prec M$. Thus $Q$ is necessarily the minimal such critical monomial. We may then apply Lemma 6.2 to $Q$ and $K$. By part (2) of Lemma 6.2 we have $B_{r-1}[N] \approx B_{r}[N] x Q$. However, $x Q>1$ and $B_{r}[N] \approx m x^{-r} N^{-r}$ by Lemma 8.3. Hence, $B_{r-1}[N] \succ$ $m x^{-r} N^{-r}$. This is a contradiction. The assertion must be true.

Now, the hypotheses of Lemma 8.5 are satisfied. Thus, there exists a function $M \in D$ with $x^{-1} \prec M \prec N$ such that $D_{r-1}[N+M]=0$. Let $L=$ $N+M$. Clearly (a) and (c) hold. It remains to prove (b). Define $\Lambda_{1}(z)=$ $\sum_{k=0}^{n} B_{k}[L] \theta^{k} z$ and $K_{1}(w)=\sum_{k=0}^{n} x^{k} B_{k}[L] w^{k}$. Let $\operatorname{trt}(L, H)=(h, q)$. Since $L \sim N$, then $m_{1}=\max \{] x^{k} B_{k}(x) L^{k}[: 0 \leqslant k \leqslant n\}$ is $\sim m$. Therefore by Lemma 8.4 applied to $L, S_{r-1}[L] \prec m_{1} x^{-r} L^{-r} \approx m x^{-r} N^{-r}$. Then, $B_{r-1}[L]$ $\prec m x^{-r} N^{-r}$.

We assert that $(h, q)<(0, r)$. Assume the contrary. By Lemma 10.3 (applied to $L), h=0$ and $q=r$. Since $q=r$, there exists a monomial $Q^{*} \in$ $\operatorname{crit}^{*}\left(K_{1}\right)$ with inst $\left(Q^{*}, K_{1}\right)=r$ and with $x^{-1} \prec Q^{*} \prec L$. Also, since $h=0$, $\Lambda_{1}$ has critical degree 0 . Hence, Lemma 10.3 implies that $K_{1}$ has exactly $r$ critical monomials $M$ with $x^{-1} \prec M \prec L$. Thus $Q^{*}$ is the only such monomial and is necessarily the minimal one. Therefore, Lemma 6.2, part (2) applied to $Q^{*}$ and $K_{1}$ gives $B_{r-1}[L] \approx B_{r}[L] x Q^{*}$. By Lemma 8.3, $B_{r}[L] \approx$ $m_{1} x^{-r} L^{-r}$ which is $\approx m x^{-r} N^{-r}$. However, since $x Q^{*}>1$, we have $B_{r-1}[L]$ $>m x^{-r} N^{-r}$. This is a contradiction. The assertion is true.

Part (b) follows from the assertion. This concludes the proof.

\section{Transform sequences.}

11.1 Definition. Let $V_{1}, V_{2}, \ldots, V_{q}$ be in $D$ with $x^{-1} \prec V_{q} \prec V_{q-1}$ $\prec \cdots \prec V_{1}$. The sequence $\left\{V_{1}, V_{2}, \ldots, V_{q}\right\}$ is called a transform sequence.

11.2 Definition. Let $\left\{V_{1}, V_{2}, \ldots, V_{q}\right\}$ be a transform sequence. Let $U_{k}=\sum_{i=1}^{k} V_{i}$ for $k=1,2, \ldots, q$. Let $\Lambda_{0}(z)=\Omega(z)$ and $K_{0}(w)=H(w)$. Let $\Lambda_{m}(z)=\sum_{k=0}^{n} B_{k}\left[U_{m}\right] \theta^{k} z$ and $K_{m}(w)=\sum_{k=0}^{n} x^{k} B_{k}\left[U_{m}\right] w^{k}$ for $m=$ $1,2, \ldots, q$. Let $\operatorname{trt}\left(V_{i}, K_{i-1}\right)=\left(r_{i}, s_{i}\right)$ for $i=1,2, \ldots, q$. If $\left(r_{i+1}, s_{i+1}\right)<$ $\left(r_{i}, s_{i}\right)$ for each $i$, then $\left\{V_{1}, V_{2}, \ldots, V_{q}\right\}$ is called a reducing transform sequence (briefly r.t.s.). If in addition $r_{q}$ is nonzero, then $\left\{V_{1}, V_{2}, \ldots, V_{q}\right\}$ is 
called a terminal r.t.s. (briefly t.r.t.s.) with terminal index $r_{q}$ (briefly t. index).

11.3 Lемма. Assume \$8.2. Then there exist t.r.t. sequences $\left\{V_{11}, V_{12}, \ldots, V_{1 k_{1}}\right\},\left\{V_{21}, V_{22}, \ldots, V_{2 k_{2}}\right\}, \ldots,\left\{V_{m 1}, V_{m 2}, \ldots, V_{m k_{m}}\right\}$ with respective $t$. indices $p_{1}, p_{2}, \ldots, p_{m}$ such that, with $U_{i}=\sum_{j=1}^{k_{i}} V_{i j}$ for $i=$ $1,2, \ldots, m$, the following hold:

(1) $U_{i} \sim N$ for $i=1,2, \ldots, m$,

(2) $\sum_{i=1}^{m} p_{i}=r$, and

(3) $x^{-1} \prec U_{i}-U_{j}$ for $1 \leqslant i, j \leqslant m$ and $i \neq j$.

Proof. The lemma is proved by induction on the multiplicity $r$. Let $r=1$. Then, $N$ is a simple critical monomial of $H$. By Lemma 10.5 , there is a function $N^{*} \in D$ with $N^{*} \sim N$ such that $\operatorname{trt}\left(N^{*}, H\right)=(s, t)<(0,1)$. Thus $s=1$ and $t=0$. Therefore, $\left\{N^{*}\right\}$ is a t.r.t.s. with t. index 1 . The lemma is valid for $r=1$.

The critical step in the proof is the reduction of the multiplicity of the critical monomials. This is accomplished through Lemma 10.5. In general, assume that for any $m<r$, for any operator $H^{*}$ of the form of $H$ with corresponding differential operator $\Omega^{*}$ of the from of $\Omega$, and for any function $M \in D$ with $x^{-1} \prec M$ and with $M \sim$ an $m$ th repeated critical monomial of $H^{*}$, there exist t.r.t. sequences which satisfy (1), (2) and (3). We need only show that the lemma is valid for $m=r$.

Let $N \in D$ be $\sim$ an $r$ th repeated critical monomial of $H$ with $x^{-1} \prec N$. By Lemma 10.5, there exists a function $N^{*} \in D$ with $N^{*} \sim N$ such that $\operatorname{trt}\left(N^{*}, H\right)=(p, q)<(0, r)$. Note that $N$ itself may suffice. Consider the polynomial $H^{*}(w)=\sum_{k=0}^{n} x^{k} B_{k}\left[N^{*}\right] w^{k}$ with corresponding differential operator $\Omega^{*}(y)=\sum_{k=0}^{n} B_{k}\left[N^{*}\right] \theta^{k} z$. By Lemma $10.3, H^{*}$ has precisely $r-p$ critical monomials $M$ with $x^{-1} \prec M \prec N^{*}$, counting multiplicity. Since $q<r$, no such monomial can have multiplicity $>r$. So by hypothesis, for each such monomial $M$ of multiplicity $m$, there exist t.r.t. sequences satisfying (1), (2) and (3) relative to $M, H^{*}$ and $\Omega^{*}$. Let the sequences be $\left\{T_{11}, T_{12}, \ldots, T_{1 a_{1}}\right\}$, $\left\{T_{21}, T_{22}, \ldots, T_{2 a_{2}}\right\} \ldots,\left\{T_{u 1}, T_{u 2}, \ldots, T_{u a_{u}}\right\}$ with respective t. indices $v_{i}$ for $i=1,2, \ldots, u$. Let $S_{i}=T_{i 1}+T_{i 2}+\cdots+T_{i a_{i}}$ for each $i$. Since distinct critical monomials $M$ of $H^{*}$ with $x^{-1} \prec M \prec N$ have differences which are $>x^{-1}$, we have

(a) $S_{i} \sim$ some $M \in \operatorname{crit}^{*}\left(H^{*}\right)$ with $x^{-1} \prec M \prec N$,

(b) $v_{1}+v_{2}+\cdots+v_{u}=r-p$ and

(c) $S_{i}-S_{j}>x^{-1}$ for $i \neq j$.

If $p>0$, then $\left\{N^{*}\right\}$ is a t.r.t.s. with t. index $p$ (for $\Omega$ ). Thus the sequences

$$
\begin{array}{r}
\left\{N^{*}\right\},\left\{N^{*}, T_{11}, T_{12}, \ldots, T_{1 a_{1}}\right\},\left\{N^{*}, T_{21}, T_{22}, \ldots, T_{2 a_{2}}\right\}, \ldots, \\
\left\{N^{*}, T_{u 1}, T_{u 2}, \ldots, T_{u a_{u}}\right\}
\end{array}
$$


satisfy the lemma. If $p=0$, then the sequences $\left\{N^{*}, T_{11}, T_{12}, \ldots, T_{1 a_{1}}\right\}$, $\left\{N^{*}, T_{21}, T_{22}, \ldots, T_{2 a_{2}}\right\}, \ldots,\left\{N^{*}, T_{u 1}, T_{u 2}, \ldots, T_{u a_{u}}\right\}$ satisfy the lemma. Parts (1), (2) and (3) clearly follow from (a), (b) and (c) above. The induction step is valid. This concludes the proof of the lemma.

\section{Independence conditions.}

12.1 Definition. Let $\left(y_{1}, y_{2}, \ldots, y_{q}\right)$ be a $q$-tuple of functions, each admissible in $F(a, b)$. Then $\left(y_{1}, y_{2}, \ldots, y_{q}\right)$ is called dependent modulo trivial functions (briefly t-dependent) in $F(a, b)$ if there is a $q$-tuple of constants $\left(k_{1}, k_{2}, \ldots, k_{q}\right)$ not all zero, such that $k_{1} y_{1}+k_{2} y_{2}+\cdots+k_{q} y_{q}$ is trivial in $F(I)$ for some open interval $I$ contained in $(a, b)$. If $\left(y_{1}, y_{2}, \ldots, y_{q}\right)$ is not $\mathrm{t}$-dependent we say it is t-independent. A t-independent family is clearly linearly independent (see [1, §9]).

12.2 Definition. Let $\left(u_{1}, u_{2}, \ldots, u_{q}\right)$ be a $q$-tuple of functions each admissible in $F(a, b)$. Let $y_{i}=\exp \int u_{i}$ for each $i$. Then $\left(y_{1}, y_{2}, \ldots, y_{q}\right)$ is called dependent modulo monomial functions (briefly m-dependent) in $F(a, b)$ if there is a $q$-tuple of constants $\left(k_{1}, k_{2}, \ldots, k_{q}\right)$ not all zero, such that $k_{1} y_{1}+$ $k_{2} y_{2}+\cdots+k_{q} y_{q}$ is either $\sim$ a complex monomial or trivial in $F(I)$ for some open interval $I$ contained in $(a, b)$. If $\left(y_{1}, y_{2}, \ldots, y_{q}\right)$ is not m-dependent, we say it is $\mathrm{m}$-independent. An m-independent family is clearly linearly independent.

12.3 Lemma. Let $c$ and $t$ be constants, $c \neq 0$ and $t>0$. Let $W$ be any function which is $\sim c x^{-1+t}$ in some $F(J)$. If $J_{1}$ is any subinterval of $J$ on which $I F(W)>0$ (respectively, $\operatorname{IF}(W)<0$ ), then for all $a$, exp $W>x^{a}$ (respectively, $\left.\exp W \prec x^{a}\right)$ in $F\left(J_{1}\right)$.

Proof. This is proved in $[3, \S 10(\mathrm{a})]$. Here $I F(W)=\cos (t \gamma+\arg c)$ for $\gamma$ in $J$. Since $I F(W)$ can have only finitely many zeros in $J$, such subintervals can be found.

12.4 LeMMA. Let $\left(W_{1}, W_{2}, \ldots, W_{q}\right)$ be a q-tuple of elements in $D$ (an $L D F_{0}$ over $F(a, b))$. Let $W_{i}>x^{-1}$ and $y_{i}=\exp \int W_{i}$ for each $i$. Assume that $W_{i}-W_{j}$ $>x^{-1}$ for any pair of indices $(i, j)$ with $i \neq j$. If $J$ is any subinterval of $(a, b)$ on which $\operatorname{IF}\left(W_{i}\right)>0$ for each $i$, then the q-tuple $\left(y_{1}, y_{2}, \ldots, y_{q}\right)$ is m-independent in $F(J)$.

Proof. The proof is by induction on $q$. Since $W_{i} \succ x^{-1}$ and $W_{i} \in D$, then $W_{i} \sim c_{i} x^{-1+d_{i}}$ for some $c_{i} \neq 0$ and $d_{i}>0$. The conclusion holds for $q=1$ by Lemma 12.3.

Assume that for any $n<q$, all $n$-tuples of functions which satisfy the hypotheses are m-independent in $F\left(J_{1}\right)$ for any open interval $J_{1} \subset J$. Assume that $\left(y_{1}, y_{2}, \ldots, y_{q}\right)$ are m-dependent. Then there exist constants $\left(k_{1}, k_{2}, \ldots, k_{q}\right)$ not all zero, such that $k_{1} y_{1}+k_{2} y_{2}+\cdots+k_{q} y_{q}=m$ in 
$F(I)$, for some open interval $I \subset J$ and where $m$ is $\sim$ a logarithmic monomial or trivial in $F(I)$. Let $I_{1}$ be any open subinterval of $I$ on which $I F\left(W_{i}-W_{j}\right)$ are nowhere zero for $i \neq j$. As in $\S 12.3$, such intervals do exist. Without loss of generality, assume that $k_{1} \neq 0$. Let

$S_{1}=\left\{i: I F\left(W_{i}-W_{1}\right)>0\right.$ on $\left.I_{1}\right\}$ and $S_{2}=\left\{i: I F\left(W_{i}-W_{1}\right)<0\right.$ on $\left.I_{1}\right\}$.

By assumption and Lemma 12.3 , for any $a$, in $F\left(I_{1}\right)$ we have $y_{i} / y_{1}>x^{a}$ for $i \in S_{1}$ and $y_{i} / y_{1} \prec x^{a}$ for $i \in S_{2}$. Since $\operatorname{IF}\left(W_{1}\right)>0$, then $\operatorname{IF}\left(-W_{1}\right)<0$ on $J$ and in $F\left(I_{1}\right), 1 / y_{1} \prec x^{a}$ for all $a$. If $S_{1}$ is empty then $k_{1}=m / y_{1}-$ $\sum_{i \in S_{2}} k_{i} y_{i} / y_{1}$. The right-hand side is trivial which implies that $k_{1}=0$ and is a contradiction. Thus, if $S_{1}$ is empty, $\left(y_{1}, y_{2}, \ldots, y_{q}\right)$ is m-independent in $F\left(I_{1}\right)$.

If $S_{1}$ is not empty, then $\sum_{i \in S_{1}} k_{i} y_{i} / y_{1}=k_{1}+m / y_{1}-\sum_{i \in s_{2}} k_{i} y_{i} / y_{1}$. Now $y_{i} / y_{1}=\exp \int\left(W_{i}-W_{1}\right)$ and $W_{i}-W_{1}>x^{-1}$. Then the functions $y_{i} / y_{1}$ for $i \in S_{1}$ satisfy the hypotheses of the lemma. The right-hand side is $\sim-k_{1}$ in $F\left(I_{1}\right)$. However, $S_{1}$ is a proper subset of $\{1,2, \ldots, q\}$. Thus by the inductive hypotheses, $k_{i}=0$ for each $i \in S_{1}$. We then write $\Sigma_{i \in S_{2}} k_{i} y_{i}+k_{1} y_{1}=m$. The set $S_{2} \cup\{1\}$ is again a proper subset of $\{1,2, \ldots, q\}$. Hence $k_{i}=0$ for all $i$. Therefore $\left(y_{1}, y_{2}, \ldots, y_{q}\right)$ is $\mathrm{m}$-independent in $F\left(I_{1}\right)$. Since the argument is valid on any such subinterval, then $\left(y_{1}, y_{2}, \ldots, y_{q}\right)$ is $\mathrm{m}$-independent in $F(J)$. This concludes the proof.

12.5 LeMMA. Let $\left(W_{1}, W_{2}, \ldots, W_{q}\right)$ be a q-tuple of elements in $D$ (an $L D F_{0}$ over $F(a, b))$. Let $W_{i}>x^{-1}$ and $y_{i}=\exp \int W_{i}$ for each $i$. For any pair of indices $(i, j)$ with $i \neq j$, assume that $W_{i}-W_{j}>x^{-1}$. Let $I F\left(W_{i}\right)>0$ on some subinterval $J$ of $(a, b)$. Let $\left(u_{i 1}, u_{i 2}, \ldots, u_{i n_{i}}\right)$ be a t-independent $n_{i}$-tuple in $F(J)$. Then the family of $u_{i j} y_{i}$ for $i=1,2, \ldots, q$ and $j=1,2, \ldots, n_{i}$ is $m$-independent in $F(J)$.

Proof. The proof is inductive on $q$. For $q=1$, assume that on some $I \subset J$, there exists an $n_{1}$-tuple of constants $\left(k_{1}, k_{2}, \ldots, k_{n_{1}}\right)$ not all zero, such that $\sum_{j=1}^{n_{1}} k_{j} u_{1 j} y_{1}=m$ where $m$ is $\sim$ a complex monomial or trivial in $F(I)$. Recall that $y_{1}=\exp \int W_{1}$ and $I F\left(W_{1}\right)>0$ on $I$. Thus by Lemma 12.3,1/y $\prec x^{a}$ in $F(I)$ for all $a$. It follows that $t=m / y_{1}$ is trivial in $F(I)$. Hence, $k_{1} u_{11}+k_{2} u_{12}$ $+\cdots+k_{n_{1}} u_{1 n_{1}}=t$ in $F(I)$. However, the $n_{1}$-tuple $\left(u_{11}, u_{12}, \ldots, u_{1 n_{1}}\right)$ is t-independent in $F(J)$. Thus $k_{j}=0$ for all $j$. The family of $u_{1 j} y_{1}$ 's is m-independent in $F(J)$. The lemma holds for $q=1$.

Assume that for any $n<q$, and for any $n$-tuple of functions $\left(y_{1}, y_{2}, \ldots, y_{n}\right)$ and corresponding $n_{i}$-tuples of $u_{i j}$ 's which satisfy the hypotheses, the result holds. Let $S=\left\{(i, j): i=1,2, \ldots, q\right.$ and $\left.j=1,2, \ldots, n_{i}\right\}$. Assume that the family $\left\{u_{i j} y_{i}:(i, j) \in S\right\}$ is m-dependent. Then there exist constants $k_{i j}$ not all zero such that $\Sigma_{s} k_{i j} u_{i j} y_{i}=m$, where $m$ is $\sim$ a complex monomial or trivial in $F(I)$ for some open subinterval $I \subset J$. Let $I_{1}$ be a subinterval of $I$ on which $I F\left(W_{i}-W_{j}\right)$ are nowhere zero for all $i$ and $j$ with 
$i \neq j$. Assume that $k_{11} \neq 0$. Let $T_{1}=\left\{i: \operatorname{IF}\left(W_{i}-W_{1}\right)>0\right.$ on $\left.I_{1}\right\}$ and $T_{2}=\left\{i: I F\left(W_{i}-W_{1}\right)<0\right.$ on $\left.I_{1}\right\}$. By assumption and Lemma 12.3, in $F\left(I_{1}\right), y_{i} / y_{1} \succ x^{a}$ for all $a$ if $i \in T_{1}, y_{i} / y_{1} \prec x^{a}$ for all $a$ if $i \in T_{2}$ and $1 / y_{1} \prec x^{a}$ for all $a$. Let $S_{m}=\left\{(i, j): i \in T_{m}\right\}$ for $m=1,2$.

If $T_{1}$ is empty, then

$$
k_{11} u_{11}+\cdots+k_{1 n_{1}}=m / y_{1}-\sum_{S_{2}} k_{i j} u_{i j} y_{i} / y_{1} .
$$

Since $u_{i j}$ is $\sim$ a complex monomial for all $i$ and $j$, the right-hand side is trivial in $F\left(I_{1}\right)$. However $\left(u_{11}, u_{12}, \ldots, u_{1 n_{1}}\right)$ is t-independent in $F(J)$. Thus $k_{1 j}=0$ for all $j$ and in particular $k_{11}=0$. This contradicts the assumptions. Hence $\left\{u_{i j} y_{i}:(i, j) \in S\right\}$ is $\mathrm{m}$-independent in $F\left(I_{1}\right)$ and thus in $F(J)$.

If $T_{1}$ is not empty, then

$$
\sum_{S_{1}} k_{i j} u_{i j} y_{i} / y_{1}=m / y_{1}-k_{11} u_{11}-\cdots-k_{1 n_{1}} u_{1 n_{1}}-\sum_{S_{2}} k_{i j} u_{i j} y_{i} / y_{1} \text {. }
$$

The right-hand side is either trivial or $\sim$ a complex monomial in $F\left(I_{1}\right)$. By assumption, the functions $y_{i} / y_{1}$ for $i \in T_{1}$ satisfy the hypotheses of the lemma. Since $T_{1}$ is a proper subset of $\{1,2, \ldots, q\}$, then by the inductive hypotheses, $k_{i j}=0$ for $(i, j) \in S_{1}$. That is, the family $\left\{u_{i j} y_{i} / y_{1}:(i, j) \in S_{1}\right\}$ is m-independent in $F(J)$. We may write

$$
\sum_{S_{2}} k_{i j} u_{i j} y_{i}+k_{11} u_{11} y_{1}+\cdots+k_{1 n_{1}} u_{1 n_{1}} y_{1}=m .
$$

However, $\{1\} \cup T_{2}$ is a proper subset of $\{1,2, \ldots, q\}$. Hence $k_{i j}=0$ for $(i, j) \in S_{2}$ or $i=1$. This is again a consequence of the inductive hypotheses. Thus $k_{i j}=0$ for all $i$ and $j$. Therefore, $\left\{u_{i j} y_{i}:(i, j) \in S\right\}$ is $\mathrm{m}$-independent in $F\left(I_{1}\right)$ and thus also in $F(J)$. This concludes the proof.

\section{Proof of Theorem 5.}

Part (1). The existence of the monomial sequence $A$ follows from Lemma 6.1. Say $A=\left\{N_{1}, N_{2}, \ldots, N_{n-p}\right\}$. This sequence has distinct monomials $N^{1}, N^{2}, \ldots, N^{s}$ of multiplicity $m_{1}, m_{2}, \ldots, m_{s}$ respectively, with $m_{1}+m_{2}$ $+\cdots+m_{s}=n-p$. By Lemma 11.3, for each $N^{j}$ of multiplicity $m_{j}$, there exist t.r.t. sequences $\{V(j, k, 1), V(j, k, 2), \ldots, V(j, k, n(j, k))\}$ with respective t. indices $p(j, k)$ for $j=1,2, \ldots, s$ and $k=1,2, \ldots, m(j)$, where $m(j)$ and $n(j, k)$ are positive integers which depend on $j$ and $(j, k)$ respectively. Define $U(j, k)=\Sigma_{r} V(j, k, r)$. Let $S=\{(j, k)$ : all $j$ and $k\}$. From Lemma 11.3 we have $U(j, k) \sim N^{j}$ for $(j, k) \in S$, and $U(j, k)-U(j, r)>x^{-1}$ for $(j, k)$ and $(j, r)$ in $S$ and $k \neq r$. Also from the same lemma, the t. indices satisfy $\Sigma_{k} p(j, k)=m_{j}$ for each $j$. Hence $\Sigma_{S} p(j, k)=n-p$. Since $N^{j}$ and $N^{k}$ are distinct when $j \neq k$, we have $N^{j}-N^{k}>x^{-1}$. Thus, $U(j, k)-U(r, t) \succ$ $x^{-1}$ if either $j \neq r$ or $k \neq t$ or both. Define $\Lambda(j, k)(z)=\sum_{r=0}^{n} B_{r}[U(j, k)] \theta^{r_{z}}$ for $(j, k) \in S$. Since each $U(j, k)$ comes from a t.r.t.s., then $\Lambda(j, k)$ has 
critical degree $p(j, k)$ for all $(j, k) \in S$. By Theorem $4, \Lambda(j, k)=0$ has, e.f.d. in $F(a, b), p(j, k)$ solutions of the form $u \sim x^{\alpha}(\log x)^{\beta}$ with $\alpha$ complex and $\beta$ a nonnegative integer. The numbers $\alpha$ and $\beta$ depend upon the coefficients of the operator $\Lambda(j, k)$. Write the $p(j, k)$ solutions as $u(j, k, r)$ for $r=$ $1,2, \ldots, p(j, k)$. This of course holds for each $(j, k) \in S$. Hence $\Omega(y)=0$ has, e.f.d. in $F(a, b), p(j, k)$ solutions $u(j, k, r) \exp U(j, k)$ for $(j, k) \in S$ and $r=1,2, \ldots, p(j, k)$. Part (1) follows by appropriately renumbering the solutions.

Part (2) is an immediate consequence of Lemmas 6.1 and 12.3.

Part (3). The existence of $\left\{M_{1}, M_{2}, \ldots, M_{p}\right\}$ and $\left\{g_{1}, g_{2}, \ldots, g_{p}\right\}$ follows readily from Theorem 4 . It remains to show that the set $\left\{g_{1}, g_{2}, \ldots, g_{p}, y_{1}\right.$, $\left.y_{2}, \ldots, y_{n-p}\right\}$ is a fundamental set of solutions on each $F(I)$ where all the functions are defined and where $I \subset(a, b)$. Since all solutions exist e.f.d. in $F(a, b)$, such intervals do exist. Let $I$ be any such interval on which the $I F\left(N_{i}\right)$ make no sign changes.

Let $Q=\{(j, k, r)$ : all $j, k$ and $r\}$. From the proof of Part (1), $\left\{g_{1}, g_{2}, \ldots, g_{p}, y_{1}, y_{2}, \ldots, y_{n-p}\right\}$ is precisely

$$
\left\{g_{1}, g_{2}, \ldots, g_{p}\right\} \cup\left\{u(j, k, r) \exp \int U(j, k):(j, k, r) \in Q\right\} .
$$

By [1, Theorem II], the $p$-tuple $\left(g_{1}, g_{2}, \ldots, g_{p}\right)$ and the $p(j, k)$-tuples $(u(j, k, 1), u(j, k, 2), \ldots, u(j, k, p(j, k)))$ for $(j, k) \in S$ are t-independent in $F(I)$.

Let $T(j, k)=\exp \int U(j, k)$ for $(j, k) \in S$. Assume that on some subinterval $I_{1}$ of $I$, there exist a $p$-tuple of constants $\left(c_{1}, c_{2}, \ldots, c_{p}\right)$ and constants $c(j, k, r)$ for $(j, k, r) \in Q$ not all zero such that

$$
c_{1} g_{1}+\cdots+c_{p} g_{p}+\sum_{Q} c(j, k, r) u(j, k, r) T(j, k)=0 \quad \text { in } F\left(I_{1}\right) .
$$

By assumption, the $I F\left(N_{i}\right)$ make no sign changes on $I_{1}$. Therefore, the $I F(U(j, k))$ make no sign changes in $I_{1}$. Define $S_{1}=\{(j, k) \in S: I F(U(j, k))$ $>0$ on $\left.I_{1}\right\}$ and $S_{2}=\left\{(j, k) \in S: I F(U(j, k))<0\right.$ on $\left.I_{1}\right\}$. Let $Q_{i}=\{(j, k, r)$ $\left.\in Q:(j, k) \in S_{i}\right\}$ for $i=1,2$. Now, $U(j, k) \sim N^{j}$ and so $U(j, k)>x^{-1}$. Differences of the $U(j, k)$ 's are $>x^{-1}$. The hypotheses of Lemma 12.4 are satisfied for $S_{1}$. Hence $\left\{T(j, k):(j, k) \in S_{1}\right\}$ is m-independent in $F\left(I_{1}\right)$. Thus by Lemma 12.5, $\left\{u(j, k, r) T(j, k):(j, k, r) \in Q_{1}\right\}$ is m-independent in $F\left(I_{1}\right)$. By Lemma 12.3, for $(j, k) \in S_{2}, T(j, k) \prec x^{a}$ in $F\left(I_{1}\right)$ for all $a$. Hence, $u(j, k, r) T(j, k) \prec x^{a}$ in $F\left(I_{1}\right)$ for all $a$ and for $(j, k, r) \in Q_{2}$. It follows that

$$
\begin{aligned}
& \sum_{Q_{1}} c(j, k, r) u(j, k, r) T(j, k) \\
&=-c_{1} g_{1}-\cdots-c_{p} g_{p}-\sum_{Q_{2}} c(j, k, r) u(j, k, r) T(j, k) .
\end{aligned}
$$


In $F\left(I_{1}\right)$, the right-hand side is either $\sim$ a monomial or trivial. Therefore, $c(j, k, r)=0$ for $(j, k, r) \in Q_{1}$. Then,

$$
c_{1} g_{1}+\cdots+c_{p} g_{p}=-\sum_{Q_{2}} c(j, k, r) u(j, k, r) T(j, k) .
$$

The right-hand side is trivial in $F\left(I_{1}\right)$. Since $\left(g_{1}, g_{2}, \ldots, g_{p}\right)$ is t-independent in $F\left(I_{1}\right)$, then $c_{i}=0$ for $i=1,2, \ldots, p$. Hence we have

$$
\sum_{Q_{2}} c(j, k, r) u(j, k, r) t(j, k)=0 .
$$

Assume that for some $(\alpha, \beta, \gamma), c(\alpha, \beta, \gamma) \neq 0$. Let $S_{3}=S_{2}-\{(\alpha, \beta)\}$ and $Q_{3}=\left\{(j, k, r) \in Q:(j, k) \in S_{3}\right\}$. Let $R(j, k)=T(j, k) / T(\alpha, \beta)$ and divide by $T(\alpha, \beta)$ to get

$$
\sum_{Q_{3}} c(j, k, r) u(j, k, r) R(j, k)=\sum_{r} c(\alpha, \beta, r) u(\alpha, \beta, r)
$$

Let $I_{2}$ be any subinterval of $I_{1}$ on which the $I F(U(j, k)-U(\alpha, \beta))$ make no sign changes for $(j, k) \in S_{3}$. Define

$$
S_{4}=\left\{(j, k) \in S_{3}: I F(U(j, k)-U(\alpha, \beta))>0 \text { on } I_{2}\right\}
$$

and

$$
S_{5}=\left\{(j, k) \in S_{3}: I F(U(j, k)-U(\alpha, \beta))<0 \text { on } I_{2}\right\}
$$

Let $Q_{i}=\left\{(j, k, r) \in Q:(j, k) \in S_{i}\right\}$ for $i=4$, 5. It follows that

$$
\begin{aligned}
\sum_{Q_{4}} c(j, k, r) u(j, k, r) R(j, k) & \\
= & -\sum_{r} c(\alpha, \beta, r) u(\alpha, \beta, r)-\sum_{Q_{s}} c(j, k, r) u(j, k, r) R(j, k) .
\end{aligned}
$$

For $(j, k) \in S_{5}$, it follows from Lemma 12.3 that $R(j, k) \prec x^{a}$ in $F\left(I_{2}\right)$ for all $a$. Hence, for $(j, k, r) \in Q_{5}, u(j, k, r) R(j, k) \prec x^{a}$ in $F\left(I_{2}\right)$ for all $a$. Thus the right-hand side is either trivial or $\sim$ a complex monomial in $F\left(I_{2}\right)$. Recall that $U(j, k)-U(\alpha, \beta)>x^{-1}$. Therefore by Lemma $12.4,\left\{R(j, k):(j, k) \in S_{4}\right\}$ is m-independent in $F\left(I_{2}\right)$. It then follows from Lemma 12.5 that $\left\{u(j, k, r) R(j, k):(j, k, r) \in Q_{4}\right\}$ is m-independent in $F\left(I_{2}\right)$. Hence we must have $c(j, k, r)=0$ for $(j, k, r) \in Q_{4}$. Upon simplification, we have

$$
\sum_{r} c(\alpha, \beta, r) u(\alpha, \beta, r)=-\sum_{Q_{s}} c(j, k, r) u(j, k, r) R(j, k) .
$$

The right-hand side is trivial in $F\left(I_{2}\right)$. Since the $p(\alpha, \beta)$-tuple $(u(\alpha, \beta, 1)$, $u(\alpha, \beta, 2), \ldots, u(\alpha, \beta, p(\alpha, \beta)))$ is t-independent in $F\left(I_{2}\right)$, then $c(\alpha, \beta, r)=$ 0 for all $r$. In particular, $c(\alpha, \beta, \gamma)=0$. This a contradiction. Hence we must have $c(j, k, r)=0$ for all $(j, k, r) \in Q_{2}$.

We have shown that $c_{j}=0$ for all $j$ and that $c(j, k, r)=0$ for all $(j, k, r)$. Hence the set $\left\{g_{1}, g_{2}, \ldots, g_{p}, y_{1}, y_{2}, \ldots, y_{n-p}\right\}$ is linearly independent in 
$F\left(I_{2}\right)$. Clearly the set must be linearly independent on any such $F\left(I_{2}\right)$. Thus on each $F(I)$, where all of the functions are defined in $F(I)$, the set is linearly independent. This completes the proof of the theorem.

\section{Remarks and examples.}

14.1 RemarK. The actual result is slightly more explicit than that of Theorem 5. In fact, if $\left\{V_{1}, V_{2}, \ldots, V_{q}\right\}$ is any t.r.t.s. with t. index $p>0$ and if $U=\Sigma V_{i}$, then e.f.d. in $F(a, b)$, the equation $\Omega(y)=0$ possesses $p$ solutions of the form $y=u \exp \int U$ where $u \sim x^{\alpha}(\log x)^{\beta}$ for some complex $\alpha$ and nonnegative integer $\beta$. The precise values of $\alpha$ and $\beta$ are given by Theorem 4 .

14.2 ExAMPLE. This example shows the necessity of Lemmas 8.5 and 10.5. Let

$$
\begin{aligned}
\Omega(y)= & x^{-3} \theta^{3} y+\left(3 x^{-5 / 2}-3 x^{-2}\right) \theta^{2} y \\
& +\left(3 x^{-1}-6 x^{-3 / 2}\right) \theta y+\left(3 x^{-1 / 2}-2 x^{-3 / 2}-x^{-2}-1\right) y .
\end{aligned}
$$

Therefore,

$$
\begin{aligned}
H(w)= & w^{3}-\left(3-3 x^{-1 / 2}\right) w^{2}+\left(3-6 x^{-1 / 2}\right) w \\
& -\left(1-3 x^{-1 / 2}+2 x^{-3 / 2}+x^{-2}\right) .
\end{aligned}
$$

Thus, $H$ has critical monomial 1 of multiplicity 3. Now, $\Omega\left(z e^{x}\right)=e^{x} \Lambda(z)$ where $\Lambda(z)=x^{-3} \theta^{3} z+3 x^{-5 / 2} \theta^{2} z+3 x^{-2} \theta z+x^{-3 / 2} z$. Let $K(w)=w^{3}+$ $3 x^{-1 / 2} w^{2}+3 x^{-1} w+x^{-3 / 2}$. Hence, $\Lambda$ has critical degree 0 and $K$ has critical monomial $-x^{-1 / 2}$ of multiplicity 3 . Then $\operatorname{trt}(1, H)=(0,3)$. By Lemma 10.5 , there exists a function $N \sim 1$ such that $\operatorname{trt}(N, H)<(0,3)$. In fact, since $-x^{-1 / 2}$ is an exact root of $K$, we may use $N=1-x^{-1 / 2}$ by Lemma 8.5. Therefore, $\Omega\left(z \exp \int N\right)=\left(\exp \int N\right) \Lambda^{*}(z)$ with $\Lambda^{*}(z)=x^{-3} \theta^{3} z-$ $(3 / 2) x^{-5 / 2} \theta z-(1 / 4) x^{-5 / 2} z$. Let $K^{*}(w)=w^{3}-(3 / 2) x^{-3 / 2} w-(1 / 4) x^{-5 / 2}$. Since $K^{*}(w)$ has critical monomials $\pm(3 / 2)^{1 / 2} x^{-3 / 4}$ and $(-1 / 6) x^{-1}$ and $\Lambda^{*}$ has critical degree $1, \operatorname{trt}(N, H)=(1,1)$. Now $F^{*}(a)=$ $-3 a / 2-1 / 4$ has root $a=-1 / 6$, so e.f.d. in $F(-\pi, \pi), \Omega(y)$ has a solution $y_{1}=g_{1} \exp N$ with $g_{1} \sim x^{-1 / 6}$.

Let $\gamma=(3 / 2)^{1 / 2}, M_{1}=N+\gamma x^{-3 / 4}$ and $M_{2}=N-\gamma x^{-3 / 4}$. Then

$$
\Omega\left(z \exp \int M_{1}\right)=\left(\exp \int M_{1}\right) \Lambda_{1}(z)
$$

with $\Lambda_{1}(z)=x^{-3} \theta^{3} z-3 \gamma x^{-11 / 4} \theta^{2} z+\left(3 x^{-5 / 2}+(3 \gamma / 4) x^{-11 / 4}\right) \theta z+$ $\left((7 / 8) x^{-5 / 2}+(\gamma / 16) x^{-11 / 4}\right) z$. Then $\operatorname{trt}\left(\gamma x^{-3 / 4}, K^{*}\right)=(1,0)$. Hence $\left\{N, \gamma x^{-3 / 4}\right\}$ is a t.r.t.s. with t. index 1 . Now $F_{1}(a)=3 a+7 / 8$ has root $a=-7 / 24$. Thus e.f.d. in $F(-\pi, \pi), \Omega(y)$ possesses a solution $y_{2}=$ $g_{2} \exp \int M_{1}$ with $g_{2} \sim x^{-7 / 24}$. Similarly,

$$
\Omega\left(z \exp \int M_{2}\right)=\left(\exp \int M_{2}\right) \Lambda_{2}(z)
$$


with $\Lambda_{2}(z)=x^{-3} \theta^{3} z-3 \gamma x^{-11 / 4} \theta^{2} z+\left(3 x^{-5 / 2}-(3 \gamma / 4) x^{-11 / 4}\right) \theta z+$ $\left((7 / 8) x^{-5 / 2}-(\gamma / 16) x^{-11 / 4}\right) z$. Thus $\operatorname{trt}\left(-\gamma x^{-3 / 4}, K^{*}\right)=(1,0)$. Since $F_{2}(a)$ $=3 a+7 / 8$ has root $a=-7 / 24$, then e.f.d. in $F(-\pi, \pi), \Omega(y)$ possesses a solution $y_{3}=g_{3} \exp \int M_{2}$ with $g_{3} \sim x^{-7 / 24}$. The set $\left\{y_{1}, y_{2}, y_{3}\right\}$ is a fundamental set of solutions for $\Omega(y)=0$.

14.3 EXAMPLE. This example demonstrates the complexity of Lemma 11.3. Consider the equation $\Omega(y)=\sum_{k=0}^{4} B_{k}(x) \theta^{k} y=0$, where $B_{0}(x)=1-13 x^{-2}$ $+29 x^{-4}-8 x^{-6}, \quad B_{1}(x)=-4 x^{-2}+26 x^{-4}-15 x^{-6}, \quad B_{2}(x)=6 x^{-4}-$ $13 x^{-6}, B_{3}(x)=-4 x^{-6}$ and $B_{4}(x)=x^{-8}$. Then $H(w)=\sum_{k=0}^{4} x^{k} B_{k}(x) w^{k}$ has critical monomial $x$ of multiplicity 4. Let $s=\exp \left(x^{2} / 2\right)$. Let $y=z s$. Then $\Omega(y)=s \Lambda(s)$ where $\Lambda(z)=\sum_{k=0}^{4} B_{k}[x] \theta^{k}$ with $B_{4}[x]=x^{-8}, B_{3}[x]=$ $B_{0}[x]=0, B_{2}[x]=-x^{-6}$ and $B_{1}[x]=x^{-6}$. Let $K(w)=\sum_{k=0}^{4} x^{k} B_{k}[x] w^{k}$. Clearly, $\Lambda$ has critical degree 2 and $K$ has critical monomials \pm 1 and $x^{-1}$. Thus $\operatorname{trt}(x, H)=(2,1)$. The critical equation for $\Lambda$ is $F(a)=-a^{2}+a$ with roots 0 and 1. Therefore, e.f.d. in $F(-\pi, \pi), \Lambda$ has solutions $g_{1} \sim 1$ and $g_{2} \sim x$ by Theorem 4 . Hence $\Omega(y)=0$ has, e.f.d. in $F(-\pi, \pi)$, solutions $y_{i}=g_{i} s$ for $i=1$, 2. Further computations give $B_{0}[x \pm 1]= \pm 6 x^{-5}+7 x^{-6}$ $\pm x^{-7}, \quad B_{1}[x \pm 1]= \pm 2 x^{-5}+13 x^{-6} \pm 4 x^{-7}, \quad B_{2}[x \pm 1]=5 x^{-6} \pm 6 x^{-7}$, $B_{3}[x \pm 1]= \pm 4 x^{-7}$, and $B_{4}[x \pm 1]=x^{-8}$. Clearly $\operatorname{trt}( \pm 1, K)=(1,0)$. The sequences $\{x, 1\}$ and $\{x,-1\}$ are t.r.t. sequences with t. indices 1 . Hence, e.f.d. in $F(-\pi, \pi), \Omega(y)=0$ has solutions $y_{3}=g_{3} s e^{x}$ and $y_{4}=g_{4} s e^{-x}$ where $g_{3} \sim x^{-3}$ and $g_{4} \sim x^{-3}$. Then $\left\{y_{1}, y_{2}, y_{3}, y_{4}\right\}$ is a fundamental set for $\Omega(y)=$ 0 .

\section{BIBLIOGRAPHY}

1. S. Bank, An asymptotic analog of the Fuchs regularity theorem, J. Math. Anal. Appl. 16 (1966), 138-151.

2. On the instability theory of differential polynomials, Ann. Mat. Pura Appl. 74 (1966), 83-112.

3. , On the asymptotic behavior of solutions near an irregular singularity, Proc. Amer. Math. Soc. 18 (1967), 15-21.

4. On nth order equations having critical degree n - 2, Ann. Mat. Pura Appl. 77 (1967), 193-206.

5. , On the structure of a fundamental set of solutions near an irregular singularity, Funkcial. Ekvac. 11 (1968), 87-100.

6. W. Strodt, Contributions to the asymptotic theory of ordinary differential equations in the complex domain, Mem. Amer. Math. Soc. No. 13 (1954).

7. , On the algebraic closure of certain partially ordered fields, Trans. Amer. Math. Soc. 105 (1962), 229-250.

8. K. Takahasi, Analytic reduction of a system of differential equations to normal form, Comment. Math. Univ. St. Paul. 22 (1973), 57-94.

Department of Mathematics, University of Dayton, Dayton, OHo 45469 